\title{
充分利用优质网络资源和教学立方平台, 助力配位化学线上教学
}

\author{
章文伟*，吕炜，黄伟 \\ 南京大学化学化工学院, 南京 210023
}

\begin{abstract}
摘要: 2020 年初, 由于受新型冠状病毒疫情影响, 学生无法按期返校开展正常教学活动。为了更好地落实教育部提出 的 “停课不停教、停课不停学” 的要求, 确保教学质量, 南京大学配位化学教学团队采用 “智慧树” 教学平台上的 “配 位化学” 慕课作为课程资源、 $Q Q$ 直播或腾讯会议作为在线授课和辅导答疑的工具、教学立方作为智慧教学平台, 成功 地开展了配位化学课程在线教学的探索和实践。该教学形式重组优化了教学资源, 实施了学生学习过程性评价和教师教 学评价, 教学效果良好。
\end{abstract}

关键词: 配位化学; 在线教学; 教学立方; 智慧树; 视频直播

中图分类号: G64; O6

\section{Making the Full Use of High Quality Network Resources and the Platform of Pedagogy Square to Facilitate Online Teaching of Coordination Chemistry}

\author{
Wenwei Zhang ", Wei Lü, Wei Huang \\ School of Chemistry and Chemical Engineering, Nanjing University, Nanjing 210023, P. R. China.
}

\begin{abstract}
In early 2020, because of the outbreak of COVID-19, students are unable to return school on time and normal teaching activities are postponed. In order to implement the requirement of "No suspension of classes" proposed by the Ministry of Education, and to ensure the teaching quality at the same time, the coordination chemistry teaching team from Nanjing University successfully carried out the exploration and practice of online teaching of coordination chemistry by using the MOOC of coordination chemistry on "Wisdom Tree" platform as the curriculum resource, QQ Live or Tencent Meeting as the online teaching tool and Pedagogy Square as the intelligent teaching platform. This teaching form reorganizes and optimizes the available resources online, and implements the student learning process evaluation and the teacher teaching evaluation. The feedback from students and teachers indicates the success of this teaching form.
\end{abstract}

Key Words: Coordination chemistry; Online teaching; Pedagogy Square; Wisdom Tree; Live Video

2019 年末至 2020 年初, 一场突如其来的新型冠状病毒肺炎 ${ }^{[1]}$ (COVID-19)引起的疫情席卷全国 乃至世界各地。受新冠肺炎疫情影响, 学生无法按期返校, 传统的线下教学活动无法正常开展。为 此, 南京大学教务处和化学化工学院在开学前一周(原教学计划)即专门组织教师进行了多次线上教 学培训和直播公开课的在线演示, 为开展线上教学积极创造条件, 努力实现教育部提出的 “停课不 停教, 停课不停学”[2,3]。

收稿: 2020-05-01; 录用: 2020-05-08; 网络发表: 2020-05-13

“通讯作者, Email: wwzhang@nju.edu.cn

基金资助: 基础学科拔尖学生培养试验计划 
南京大学配位化学课程是一门本、硕贯通课程, 教学对象是本科三年级和硕士一年级学生。由 于配位化学是一门处于不断发展和完善中的专业课程, 其内涵和外延尚在不断丰富和拓展中, 其授 课对象既有高年级本科生, 又有研一学生, 因此, 我们希望本课程能够将配位化学的理论知识和最 新研究前沿完美地结合, 帮助学生尽快完成从课堂教学到科研实践的过渡, 实现从理论学习到科研 创新的转变。由于受疫情影响无法开展面对面线下教学, 如何利用现有的网络资源和教学平台进行 线上教学, 确保线上教学与线下教学等质同效? 为此, 我们通过以下方式, 对配位化学线上教学进 行了积极的探索和实践, 取得了良好的效果。

\section{1 充分利用优质网络资源和智慧教学平台, 重组教学资源、优化教学过程}

配位化学通常是一门选修课, 很多高校本科阶段并不开设配位化学课程(不少高校开设中级无机 化学课程, 里面会介绍一些配位化学相关知识)。就我们所知, 目前为止, 关于配位化学的教材并不 多见 ${ }^{[4-11]}$, 在线教学资源如慕课、在线精品开放课程等则更是少之又少。所幸, 无机化学界的有识 之士看到了问题所在。由兰州大学刘伟生教授牵头, 南开大学卜显和教授、南京大学孙为银和左景 林教授等一大批高校知名学者, 共同参与制作了适合本科生和研究生教学的配位化学慕课, 于 2017

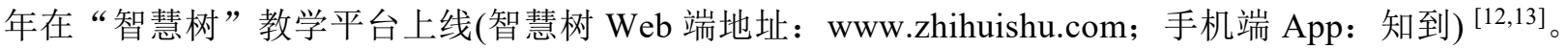
这些优质的网上教学资源对配位化学教学发展和质量提升客观上起到了很好的促进和推动作用。如 何利用这些已有的线上优质资源, 为在线教学服务, 确保线上教学学生的学习效果与线下教学等质 同效, 甚至更好? 为此, 我们结合开学前学校组织的多次线上教学培训的收获和自身对各平台的熟 悉情况, 采用师生都较为熟悉的 $\mathrm{QQ}$ 直播或腾讯会议进行线上教学, 以最大程度解决师生面对面无 障碍交流途径被切断所产生的影响。在此基础上, 将 “智慧树” 网的配位化学慕课和 “教学立方过 程化教学与学业评价平台” (简称教学立方)相结合, 对教学资源进行重组, 实施学生学习的过程性 评价和教师教学过程的质量监控, 优化教学过程, 提升教学质量。具体如图 1 所示。

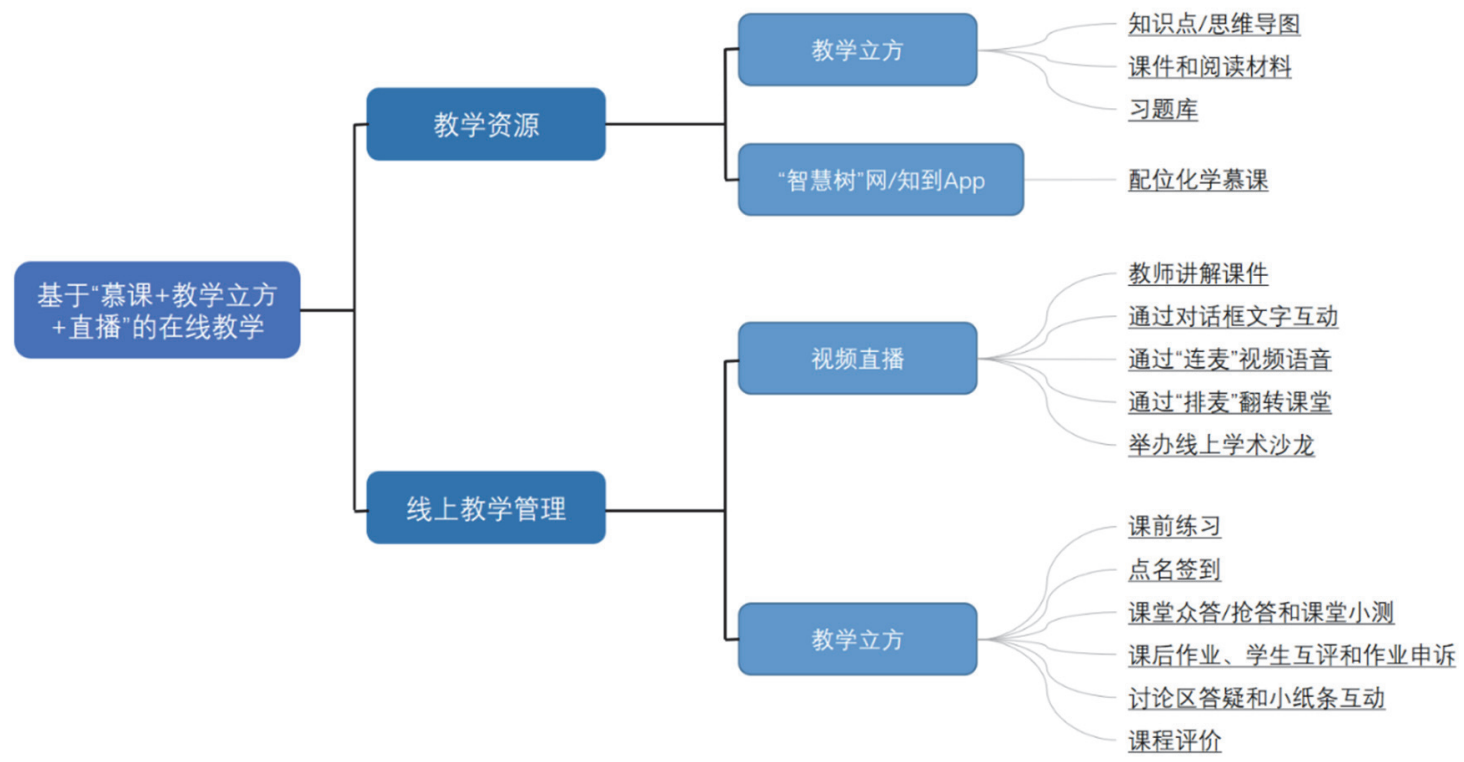

图 1 基于 “慕课 + 教学立方 + 直播” 的配位化学在线教学

\section{1 通过教学立方和慕课, 完善配位化学教学资源, 精心设计教学方案和教学内容}

首先建立班级 QQ 群, 同时在教学立方上建立配位化学课程, 生成课程邀请码, 学生通过 QQ 群号和课程邀请码进入相应班级和课程。QQ 群用于发布通知和进行线上交流, 亦可在线回答学生 
问题; 教学立方有签到、课前练习、课堂小测、作业、课件、教学分析、教学评价和沟通等多种功 能用来辅助教学, 实现教学的过程化管理和质量监控。

由于配位化学课程是一门本、硕贯通课程, 参与学习的对象不仅有本科生, 还有研究生, 考虑 到学生的配位化学基础可能不尽相同, 为此在开课前, 我们利用教学立方发布了一份简单的调查问 卷, 调查选修本门课程的研究生在本科期间是否上过 “配位化学” 或类似课程(比如: 中级无机化 学), 以便了解学生的课程基础, 制定合理的教学计划和方案。问卷调查结果显示, $79.5 \%$ 的学生并 没有配位化学相关基础知识。因此, 配位化学基本知识基础理论的讲解需要着重加强。但是, 尽管 如此, 我们还是要清楚地认识到, 我们的培养目标是要帮助学生尽快完成从课堂教学到科研实践的 过渡, 实现从理论学习到科研创新的转变。因此, 基础理论知识的学习固然重要, 但是学习的最终 目的是要将理论知识应用于实际的科学研究中。为此, 在我们的教学中, 一定要实时引入最新研究 成果, 将基础理论和研究前沿相结合, 加深学生对基础理论的理解, 使学生能够将理论与实践融会 贯通。例如, 在讲授配位化学前沿领域之一的 “超分子化学与晶体工程” 专题报告时, 针对超分子 合成子的设计过程中, 如何合理利用配位化学的基本原理来规划配体(电子效应、几何效应、对称性、 手性、配位原子的种类和数目等)和随后的金属配位反应(中心金属离子种类和电荷的选择、阴离子 效应、反应条件的控制、多种弱相互作用的协同等)这一问题, 结合文献和授课教师的个人研究体会

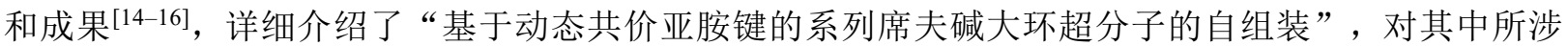
及的各种超分子作用力 (经典和非经典氢键、芳环 $\pi-\pi$ 、金属/非金属 $-\pi$ 相互作用等) 以及展现出的相 关功能(阴离子识别、选择性气体吸附、苂光传感、稀土敏化发光、光敏薄膜与半导体、抗菌活性等) 做了细致的讲解。

开课前, 我们还提前和 “智慧树” 网的老师进行沟通, 将学生信息导入, 发布通知告知学生, 让学生自行注册通过 “知到” App 进行在线学习。学生可以根据自己的基础, 选择本科生版或研究 生版慕课进行自主学习。“配位化学” 慕课作为学生课前预习和课后复习的在线资源, 与我们的线 上授课相辅相成, 有利于知识的巩固和提高。

此外, 为组织好线上教学, 我们还在教学立方平台提前做好以下六项工作: (1) 开学前一周即公 布教学计划, 便于学生提前规划学习进度; (2) 绘制每一章节的知识树, 给出思维导图, 强调知识 点, 便于学生学习时抓住重点(图 2); (3) 提前发布课件和相关学习资料, 课件关联相关知识点, 学 习资料则是和主要知识点相关的、较为新颖的科研文献资料, 以利于学生课前预习, 掌握重点, 拓 展知识面, 了解科研前沿和发展动态; (4) 提前设计和发布课前练习题, 督促学生课前预习, 检测学 生预习效果。课前练习通常设置成上课前半天结束, 以便教师根据课前练习统计分析结果, 了解学 生知识掌握情况, 做到线上授课更加有的放矢, 将学生未掌握的难点知识做更为细致深入的讲解; (5) 提前设计好课堂小测, 促进学生线上集中注意力听课, 调节课堂气氛, 检测听课效果; (6) 提前 录入课后作业, 并预设好发布时间(通常设置为下课时间节点), 以便检测学生知识掌握情况。通常, 课前练习-课堂小测-课后作业的难度是逐级增大的, 分别对应于课前预习-课堂听课-课后复习, 经 过这样一个学习和消化的过程, 学生对知识的理解和掌握逐渐深入, 符合教育教学规律。通过上述 “六个提前”, 对每一堂进行精心设计, 确保每一堂课的教学质量。

\section{2 基于 $Q Q$ 直播和教学立方, 优化在线教学过程}

通常, 在课前 $15 \mathrm{~min}$ 左右, 教师会在 $\mathrm{QQ}$ 群中提醒学生准备上课, 同时进入 $\mathrm{QQ}$ 直播间或腾讯 会议室, 做前期准备并调试直播设备。在学生都进入直播间或会议室后, 教师会询问学生音视频是 否正常, 待一切正常后, 即开始通过屏幕共享的方式进行线上授课。直播过程中, 学生如果有疑问 可以直接提问, 教师或助教可以在线回答。在课程进行期间, 教师可挑选适当时间利用教学立方进 行签到、课堂小测、抢答和众答等互动活动, 查看学生到课情况和学习情况, 并根据教学立方统计 给出的即时教学统计分析结果, 进行更加有针对性的讲解。根据教学立方记录下来的学生历次课堂 表现, 给出课程学习平时分。每一堂课结束前, 教师还会连麦学生, 与学生进行互动, 询问学生哪 
些地方不太明白或者还有疑问, 及时进行答疑解惑。通过 “直播 + 教学立方” 这样一种模式, 可以 即时做到学生学习的过程性评价和教师教学过程的质量监控, 优化教学过程, 提升教学质量。以下 我们将通过具体案列进行说明。

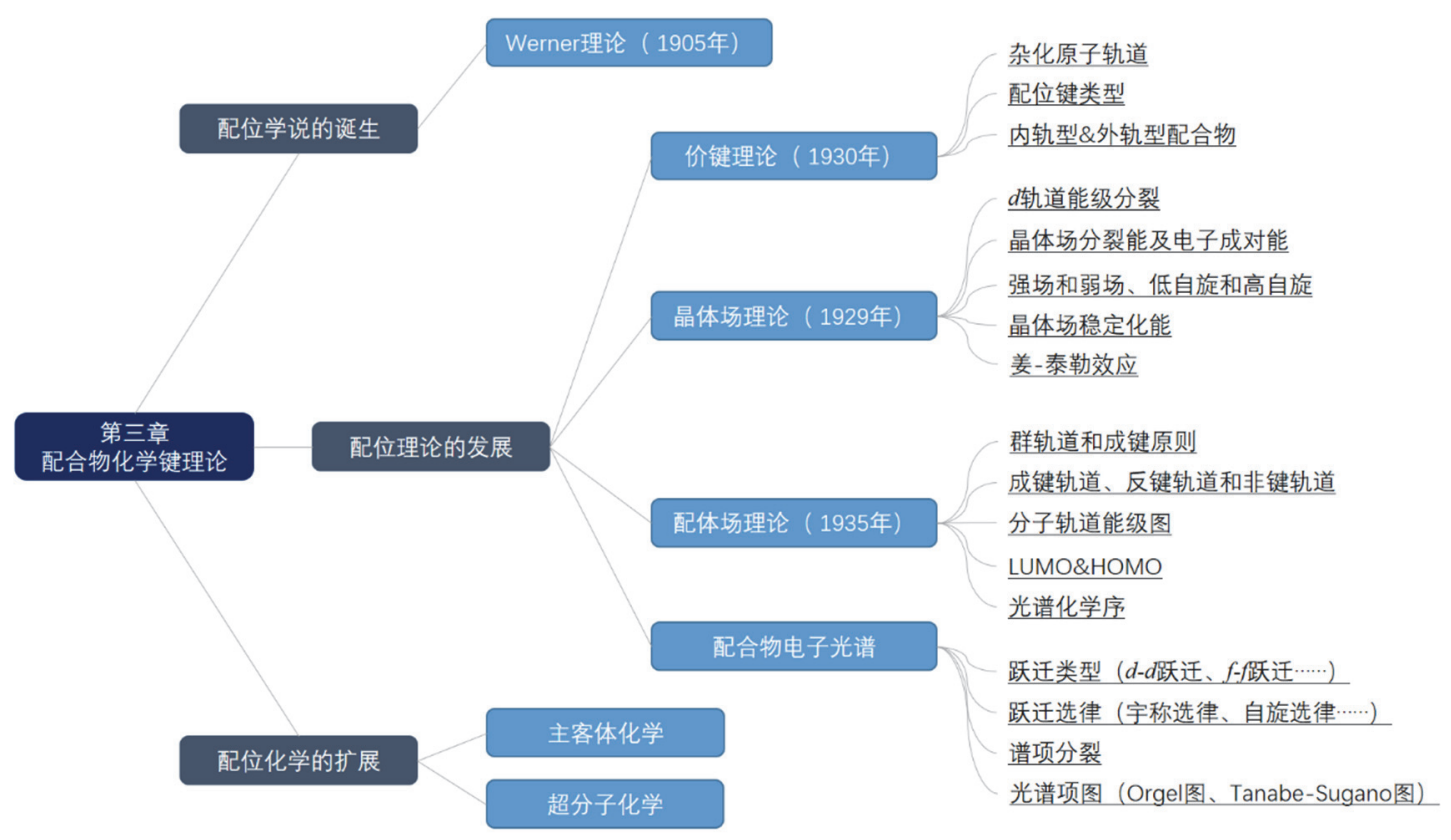

图 2 知识树示例

由于在课前，教师已经预先将课件、相关参考资料和文献上传至教学立方平台，供学生预习, 因此通过教学立方的阅读记录, 教师可以很轻松地掌握学生的预习情况。例如, 图 3 给出了学生对 “配合物的基本概念” 这一章课件的阅读情况。通过课件阅读分析, 我们知道所有学生课前都对此 课件进行了阅读，并且在第 3、7-9、28 页所花时间较多，这些内容和配体的分类、成键方式、配合 物的命名相关, 说明绝大多数学生需要花费一定的时间来对这些内容进行理解和吸收, 因此上课的 时候就需要教师对这些知识进行重点讲解。为此在课堂上, 我们对这些概念和知识进行了较为详细

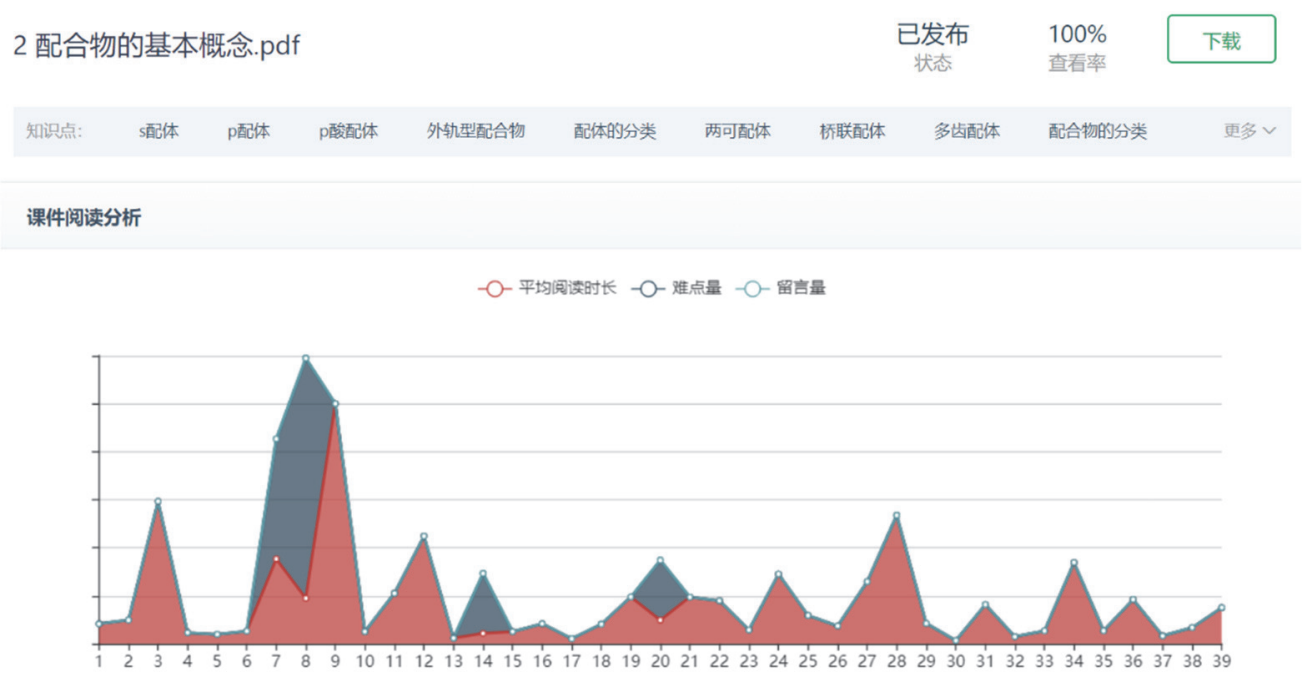

图 3 课件阅读分析 
立方的使用, 可以将阅读资料统一上传到课件目录中, 并与相关知识点进行关联, 既方便了教师对 文献资料的管理, 也让学生对需要阅读的文献资料和课堂所学知识进行有效衔接, 有利于理论联系 实践。每学期的第一堂绪论课, 我们便会告知学生, 除了理论考试, 课程还要求学生查阅文献, 根 据自己感兴趣的研究主题, 提交学期论文。本学期, 为了检验学生对理论知识的掌握和文献资料的 理解, 我们打算在学期最后, 借助于 $\mathrm{QQ}$ 直播或腾讯会议平台, 组织 1-2 次在线课堂学术沙龙, 由 学生阐述自己提交的学期论文的主要内容, 其中关联了哪些主要的配位化学理论知识, 所阅读的文 献的科学思想精髓体现在何处。教师和其他同学均可参与讨论并进行点评。通过这样一种类似于翻 转课堂的学术交流，将有利于学生理论知识和科研实践的融会贯通。

\section{3 通过教学立方, 及时跟踪学生学习效果并进行交流反馈}

为了帮助学生更好地理解课堂知识, 我们利用教学立方发布课后作业, 并设置提交日期, 督促 学生及时完成。

学生完成作业后, 我们采用学生互批作业的方式(图 5), 让学生对照答案进行批改(提前将答案 和试题分析录入系统中, 每份作业将随机发给两位学生进行批改), 加深对相关知识的理解, 并统计 作业提交情况和完成情况, 给出作业平时分。如果有学生在规定时间内忘记批改, 未批改的作业则 由教师进行批改并给出分数。

在批改作业的过程中, 学生对相互批改的结果如有异议, 可以通过教学立方提出申诉, 教师会 及时查看并进行解答, 确保学生能够及时获得反馈(图 5)。有争议的批改, 最终得分由教师评定, 这 可以纠正学生互批中的疏漏, 也保证了最终成绩评定的公平性。

最后, 我们会根据教学立方对习题正确率的统计数据, 挑选出错率较高的习题进行详细讲解, 帮学生理解重点、难点知识。

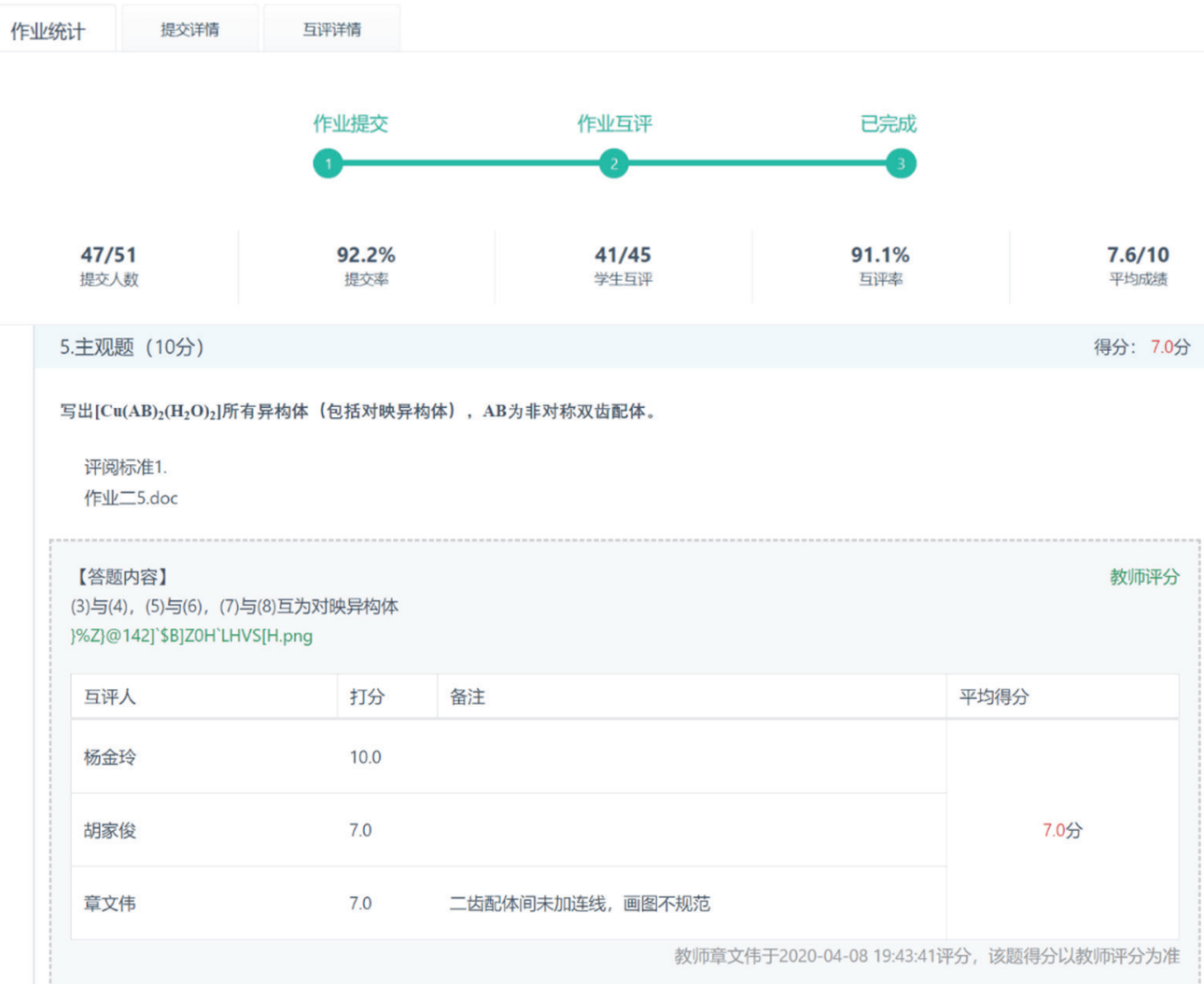

图 5 作业互批和申诉反馈 
此外, 学生在学习过程中遇到问题, 可以在讨论区发帖或通 过小纸条向教师询问, 寻求帮助。教师通过答疑解惑, 及时帮助 学生解决问题, 促进学生知识掌握(图 6)。教学立方讨论区这一 模块很好地解决了课后师生互动交流的需求。

\section{2 教学成效}

经过大半个学期的教学实践, 我们发现, 基于 “慕课 + 教学 立方 + 直播” 这样一种多维度教学模式, 通过将优质的网络资源 和智慧型教学平台相结合，提前对教学过程进行精心设计，可以 将教学资源进行有效重组, 优化教学过程, 实现教学过程的精细 化管理, 充分调动学生的学习主动性和积极性, 保证课程教学质 量(图 7)。我们欣喜地发现, 由于使用教学立方对教学过程进行 了合理设计, 特别是课前练习和课堂小测的实施, 虽然我们对 “智慧树” 网配位化学慕课的学习未做硬性规定, 但是, 学生主 动学习的热情很高, 有 $88.7 \%$ 的学生学习了研究生版慕课, $23.2 \%$ 的学生学习了本科生版慕课(学生可以根据自身基础和学习能 力, 学习不同版本, 亦可以两者都学习), 学习比率明显高于去年

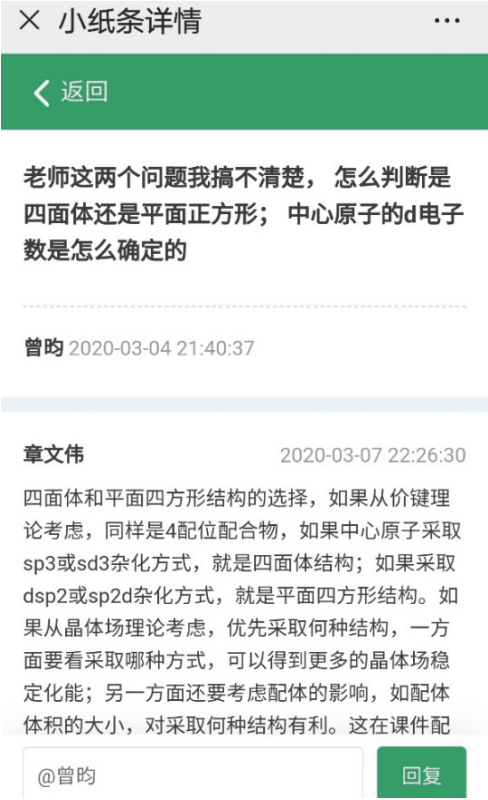

图 6 交流讨论 同一时期未使用教学立方进行教学管理的数据(2019 年这一比率分别是 34.9\%和 7.0\%)。由此可见, 合理的教学设计可以有效促进学生自主学习, 最大程度发挥网络资源共享作用。

\begin{tabular}{ccccc|crrrr} 
课程学生数量 & 罢计授课次数 & 发起互动次数 & 学生响应次数 & 课程综合评分 & 知识掌握率 & 签到到淉率 & 作业完成率 & 课堂活跃率 & 综合活跃率 \\
5 & 17 & 53 & 1572 & 4.85 & $65.7 \%$ & $90.1 \%$ & $89.1 \%$ & $88.3 \%$ & $83.3 \%$
\end{tabular}

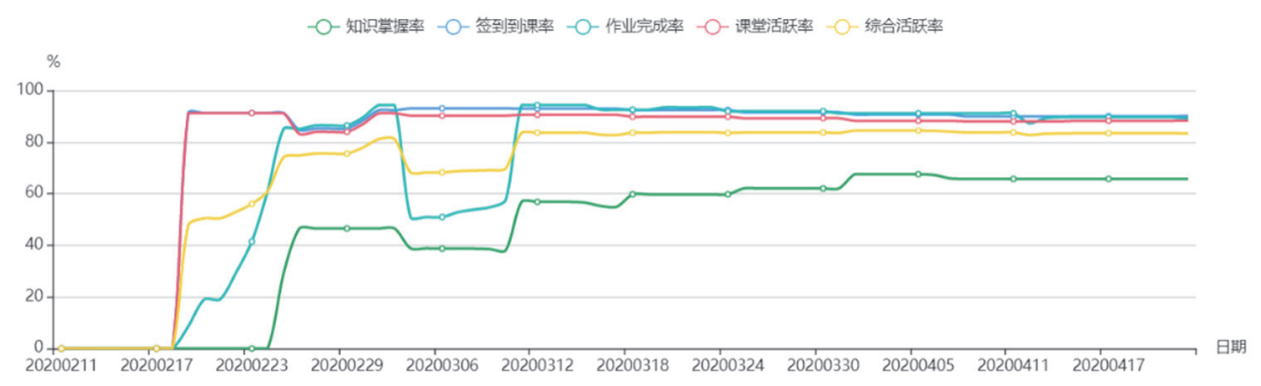

图 7 课程教学数据报告

\section{3 总结与展望}

经过对配位化学在线教学的精心准备和合理设计, 基于智慧树、QQ 直播/腾讯会议和教学立方 三结合的在线教学形式, 重组了教学资源, 优化了教学过程。该方案能够有效促进学生自主学习, 让教师全程把握学生学习及授课过程, 及时了解学生对知识的掌握程度, 高效完成与学生的互动, 实现学生学习的过程性评价和教师教学评价, 获得了学生认可。随着在线教学的持续深入开展, 借 助现代信息技术，线上教学、线上线下混合式教学必将成为今后教学发展的一种趋势。 
[2] 教育部关于 2020 春季学期延迟开学的通知. [2020-01-27]. http://www.moe.gov.cn/jyb_xwfb/gzdt_gzdt/s5987/202001/t20200127_416672.html.

[3] 教育部: 利用网络平台, “停课不停学”. [2020-01-29]. http://www.moe.gov.cn/jyb_xwfb/gzdt_gzdt/s5987/202001/t20200129_416993.html.

[4] 戴安邦. 配位化学. 北京: 科学出版社, 1987.

[5] 罗勤慧. 配位化学. 北京: 科学出版社, 1987.

[6] 孙为银. 配位化学. 第 1 版. 北京: 化学工业出版社, 2004

[7] 章慧, 等. 配位化学——原理与应用. 北京: 化学工业出版社, 2009.

[8] 孙为银. 配位化学. 第 2 版. 北京: 化学工业出版社, 2010 .

[9] 罗勤慧, 等. 配位化学. 北京: 科学技术出版社, 2012 .

[10] 刘伟生. 配位化学. 北京: 化学工业出版社, 2013.

[11] 刘伟生，卜显和. 配位化学. 第 2 版. 北京: 化学工业出版社, 2019.

[12] 配位化学-研究生版-智慧树网. [2020-02-11]. https://coursehome.zhihuishu.com/courseHome/2067613\#teachTeam.

[13] 配位化学-本科生版-智慧树网. [2020-02-11]. https://coursehome.zhihuishu.com/courseHome/2067615\#teachTeam.

[14] Meyer, C. D.; Joiner, C. S.; Stoddart, J. F. Chem. Soc. Rev. 2007, 36, 1705.

[15] Nitschke, J. R. Angew. Chem., Int. Ed. 2014, 53, 10701.

[16] Zhang, L.; Xiang, L.; Hang, C.; Liu, W.; Huang, W.; Pan, Y. Angew. Chem., Int. Ed., 2017, 56, 7787. 